\title{
On the Betti numbers of some classes of binomial edge ideals
}

\author{
Sohail Zafar \\ Mathematics Department \\ University of Management and Technology \\ Lahore, Pakistan \\ sohailahmad04@gmail.com \\ Zohaib Zahid* \\ Abdus Salam School of Mathematical Sciences \\ Government College University \\ Lahore, Pakistan \\ zohaib_zahid@hotmail.com
}

Submitted: Sep 3, 2013; Accepted: Dec 13, 2013; Published: Dec 20, 2013

Mathematics Subject Classifications: 05E40, 16E30

\begin{abstract}
We study the Betti numbers of binomial edge ideal associated to some classes of graphs with large Castelnuovo-Mumford regularity. As an application we give several lower bounds of the Castelnuovo-Mumford regularity of arbitrary graphs depending on induced subgraphs.
\end{abstract}

Keywords: Castelnuovo-Mumford regularity, Betti numbers, binomial edge ideal.

\section{Introduction}

Let $K$ denote a field. Let $G$ denote a connected, simple and undirected graph over the vertices labeled by $[n]=\{1,2, \ldots, n\}$. The binomial edge ideal $J_{G} \subseteq S=K\left[x_{1}, \ldots, x_{n}, y_{1}, \ldots\right.$ ,$\left.y_{n}\right]$ is an ideal generated by all binomials $x_{i} y_{j}-x_{j} y_{i}, i<j$, such that $\{i, j\}$ is an edge of $G$. It was introduced in [4] and independently at the same time in [6]. It is a natural generalization of the notion of monomial edge ideal which is introduced by Villarreal in $[12]$.

${ }^{*}$ Research supported by HEC Pakistan. 
The main purpose of this paper is to study the minimal free resolution of certain classes of binomial edge ideals. The arithmetic properties of binomial edge ideals in terms of combinatorial properties of graphs (and vice versa) were studied by many authors in [4], [6], [3], [13], [11], [7], [8], [5], and [9]. The reduced Gröbner basis and minimal primary decomposition of binomial edge ideal was given in the paper of Herzog et al. [4]. The Cohen-Macaulay property of binomial edge ideal were studied in [3], [7] and [8]. As a certain generalization of the Cohen-Macaulay property the second author has studied approximately Cohen-Macaulay property in [13].

There is not so much work done so far in the direction of the Betti numbers and Castelnuovo-Mumford regularity of binomial edge ideals. The minimal free resolution of the binomial edge ideal of simplest classes (complete graph and line graph) is well known. In [9], the authors determine the initial Betti number of the binomial edge ideal of an arbitrary graph. In fact they shows that $\beta_{2,3}=2 l$ where $l$ is the total number of 3-cycles in the graph $G$. They also discussed the vanishing and non-vanishing of certain Betti numbers. In [11], there is a computation of the Castelnuovo-Mumford regularity and all the Betti numbers in the case of complete bipartite graph. The relationship between the Betti numbers of a graph and the Betti numbers of its induced subgraph (see Theorem 8) was recently shown in [5] by Matsuda and Murai. They also give the CastelnuovoMumford regularity bounds for binomial edge ideals, namely $\ell-1 \leqslant \operatorname{reg}\left(S / J_{G}\right) \leqslant n-1$, where $\ell$ denotes the number of vertices of the longest induced line graph of $G$.

In the present paper we compute the Castelnuovo-Mumford regularity and all the Betti numbers in the case of cycle graphs and two more classes of graphs which we denote by $\mathcal{T}_{3}$ and $\mathcal{G}_{3}$ (see Definitions 22 and 25). In all of our classes Castelnuovo-Mumford regularity is quite large. As an application of our investigation we improve the lower bounds for the Castelnuovo-Mumford regularity of an arbitrary graph by applying as it was done in [5].

The paper is organized as follows: In Section 2, we introduce some notation and give some results that we need in the rest of the paper. In particular we give a short summary on minimal free resolutions. In Section 3, we compute the Castelnuovo-Mumford regularity and the Betti numbers of the binomial edge ideal associated with a cycle graph and obtain a lower bound for the Castelnuovo-Mumford regularity of an arbitrary graph. In Section 4, we do the same for the classes of graphs $\mathcal{T}_{3}$ and $\mathcal{G}_{3}$ as we did for the cycle in Section 3.

\section{Preliminaries}

In this section we will introduce the notation used in the article. Moreover we summarize a few auxiliary results that we need.

We denote by $G$ a connected undirected graph on $n$ vertices labeled by $[n]=\{1,2, \ldots$, $n\}$. For an arbitrary field $K$ let $S=K\left[x_{1}, \ldots, x_{n}, y_{1}, \ldots, y_{n}\right]$ denote the polynomial ring in the $2 n$ variables. To the graph $G$ one can associate an ideal $J_{G} \subset S$ generated by all binomials $x_{i} y_{j}-x_{j} y_{i}$ for $i<j$ such that $\{i, j\}$ forms an edge of $G$. This Ideal $J_{G}$ is called binomial edge ideal associated to the graph $G$. This construction was invented 
by Herzog et al. in [4] and independently found in [6]. At first let us recall some of their definitions.

Definition 1. For a subset $W \subset[n]$, a graph $G_{W}$ on vertex set $W$ is called induced subgraph of $G$ if for all $i, j \in W,\{i, j\}$ is an edge of $G_{W}$ if and only if $\{i, j\}$ is an edge of G.

Definition 2. Fix the previous notation. For a set $T \subset[n]$, let $G_{[n] \backslash T}$ is the induced subgraph of $G$ with vertex set $[n] \backslash T$. Let $c=c(T)$ denote the number of connected components of $G_{[n] \backslash T}$. Let $G_{1}, \ldots, G_{c}$ denote the connected components of $G_{[n] \backslash T}$. Then define

$$
P_{T}(G)=\left(\cup_{i \in T}\left\{x_{i}, y_{i}\right\}, J_{\tilde{G}_{1}}, \ldots, J_{\tilde{G}_{C(T)}}\right),
$$

where $\tilde{G}_{i}, i=1, \ldots, c$, denotes the complete graph on the vertex set of the connected component $G_{i}, i=1, \ldots, c$.

The following result whose proof can be found in Section 3 of the paper [4] is important for the understanding of the binomial edge ideal of $G$.

Lemma 3. With the previous notation the following holds:

(a) $P_{T}(G) \subset S$ is a prime ideal of height $n-c+|T|$, where $|T|$ denotes the number of elements of $T$.

(b) $J_{G}=\cap_{T \subseteq[n]} P_{T}(G)$.

(c) $J_{G} \subset P_{T}(G)$ is a minimal prime if and only if either $T=\emptyset$ or $T \neq \emptyset$ and $c(T \backslash\{i\})<c(T)$ for each $i \in T$.

Therefore $J_{G}$ is the intersection of prime ideals. That is, $S / J_{G}$ is a reduced ring. Moreover, we remark that $J_{G}$ is an ideal generated by quadrics and therefore homogeneous, so that $S / J_{G}$ is a graded ring with natural grading induced by the $\mathbb{N}$-grading of $S$.

Remark 4 . If we define a grading on $S$ by setting $\operatorname{deg} x_{i}=\operatorname{deg} y_{i}=e_{i}$ where $e_{i}$ is the $i$-th unit vector of $\mathbb{N}^{n}$ then $S / J_{G}$ is $\mathbb{N}^{n}$-graded too.

Let $M$ a graded finitely generated $S$-module. By Hilbert's Syzygy Theorem, $M$ has a finite minimal graded free resolution:

$$
F_{\bullet}: 0 \rightarrow F_{p} \rightarrow \cdots \rightarrow F_{1} \rightarrow F_{0} \rightarrow M \rightarrow 0
$$

where $F_{i}=\bigoplus_{j} S\left(-d_{i, j}\right)^{\beta_{i, j}}$ for $i \geqslant 0$ and $p$ is called the projective dimension of $M$. The numbers $\beta_{i j}$ are uniquely determined by $M$ i.e. $\beta_{i, j}(M)=\operatorname{dim}_{K} \operatorname{Tor}_{i}^{S}(K, M)_{j}, i, j \in \mathbb{Z}$, as graded Betti numbers of $M$. We can also define Castelnuovo-Mumford regularity $\operatorname{reg} M=\max \left\{j-i \in \mathbb{Z} \mid \beta_{i, j}(M) \neq 0\right\}$. The Betti table looks as in the following:

\begin{tabular}{c|cccc} 
& 0 & 1 & $\cdots$ & $p$ \\
\hline 0 & $\beta_{0,0}$ & $\beta_{1,1}$ & $\cdots$ & $\beta_{p, p}$ \\
1 & $\beta_{0,1}$ & $\beta_{1,2}$ & $\cdots$ & $\beta_{p, p+1}$ \\
$\vdots$ & $\vdots$ & $\vdots$ & $\vdots$ & \\
$r$ & $\beta_{0, r}$ & $\beta_{1,1+r}$ & $\cdots$ & $\beta_{p, p+r}$
\end{tabular}


Note that all the $\beta_{i, j}$ outside of the Betti table are zero. For more details and related facts we refer the book of Burns and Herzog [1]. The following result in [1] is important for us.

Lemma 5. Let $M$ denote a finitely generated graded $S$-module then Hilbert series of $M$ can be computed from the graded Betti numbers as follows:

$$
H(M, t)=\frac{\sum_{-\infty<j<\infty} \sum_{i=0}^{2 n}(-1)^{i} \beta_{i, j}(M) t^{j}}{(1-t)^{2 n}} .
$$

Definition 6. Let $M$ denote a finitely generated graded $S$-module and $d=\operatorname{dim} M$. For an integer $i \in \mathbb{Z}$ put

$$
\omega^{i}(M)=\operatorname{Ext}_{S}^{2 n-i}(M, S(-2 n))
$$

and call it the $i$-th module of deficiency. The module $\omega(M):=\omega^{d}(M)$ is called the canonical module of $M$.

These modules have been introduced and studied in [10]. The following theorem whose proof can be found in [1, Corollary 3.3.9] is important for us.

Theorem 7. Let $M$ denote a finitely generated graded Cohen-Macaulay $S$-module of projective dimension $p=2 n-\operatorname{dim} M$. Let

$$
F_{\bullet}: 0 \rightarrow F_{p} \rightarrow \cdots \rightarrow F_{1} \rightarrow F_{0} \rightarrow 0
$$

be the minimal free resolution of $M$. Let $G_{\bullet}=\operatorname{Hom}_{S}\left(F_{\bullet}, S(-2 n)\right)$ be the dual complex

$$
G_{\bullet}: 0 \rightarrow G_{p} \rightarrow \cdots \rightarrow G_{1} \rightarrow G_{0} \rightarrow 0
$$

where $G_{i}=\operatorname{Hom}\left(F_{p-i}, S(-2 n)\right)$ for $i=0, \ldots, p$. Then $G_{\bullet}$ is the minimal free resolution of $\omega(M)$.

Recently K. Matsuda and S. Murai in [5, Corollary 2.2] proved the relationship between the Betti numbers of the graph with the Betti numbers of its induced subgraph. The result is as follows:

Theorem 8. Let $G_{W}$ be an induced subgraph of $G$. Then $\beta_{i, j}\left(S / J_{G}\right) \geqslant \beta_{i, j}\left(S / J_{G_{W}}\right)$ for all $i, j$.

\section{Betti Numbers of the binomial edge ideal of a cycle}

Definition 9. A cycle is a graph in which all the vertices are of degree 2 .

In particular, for $n=3$ it is triangle and $n=4$ it is square. We denote the cycle on vertex set $[n]$ by $C$ and its binomial edge ideal by $J_{C}$. It is known from [13, Theorem 4.5] that, $S / J_{C}$ is approximately Cohen-Macaulay ring of $\operatorname{dim}\left(S / J_{C}\right)=n+1$. 
Theorem 10. Let $J_{L}$ be the binomial edge ideal of a line $L$ on the vertex set $[n], g=$ $x_{1} y_{n}-x_{n} y_{1}$ and $J_{\tilde{G}}$ be binomial edge ideal of the complete graph on $[n]$ then:

(a) $J_{L}: g / J_{L} \cong \omega\left(S / J_{\tilde{G}}\right)(2)$

(b) The Hilbert series of $S / J_{C}$ is

$$
H\left(S / J_{C}, t\right)=\frac{1}{(1-t)^{n+1}}\left((1+t)^{n-1}-t^{2}(1+t)^{n-1}+(n-1) t^{n}+t^{n+1}\right) .
$$

One might see the proof of the above Theorem in [13, Lemma 4.8 and Theorem 4.10]. In order to compute the Betti numbers of a cycle we need to understand the modules $J_{L}: g / J_{L}$ and $S / J_{L}: g$. To this end we need the following lemma about the canonical module of $S / J_{L}: g$.

Lemma 11. With the notation as before we have

(a) $\omega\left(S / J_{L}: g\right) \cong J_{\tilde{G}} / J_{L}(-2)$.

(b) The minimal number of generators of $\omega\left(S / J_{L}: g\right)$ is $\left(\begin{array}{c}n-1 \\ 2\end{array}\right)$.

Proof. From Theorem 10 we have $J_{L}: g / J_{L} \cong \omega\left(S / J_{\tilde{G}}\right)(2)$. Now consider the exact sequence

$$
0 \rightarrow \omega\left(S / J_{\tilde{G}}\right)(2) \rightarrow S / J_{L} \rightarrow S / J_{L}: g \rightarrow 0 .
$$

All modules in above exact sequence are Cohen-Macaulay of dimension $n+1$. By applying local cohomology and dualizing it we get the following exact sequence

$$
0 \rightarrow \omega\left(S / J_{L}: g\right) \rightarrow S / J_{L}(-2) \rightarrow S / J_{\tilde{G}}(-2) \rightarrow 0 .
$$

Which implies the isomorphism in (a) and then (a) gives us (b).

All Tor modules of $J_{L}: g / J_{L}$ are given in the following lemma.

Lemma 12. We have the following isomorphisms.

(a) $\operatorname{Tor}_{i}^{S}\left(K, J_{L}: g / J_{L}\right) \cong K^{c_{i}}(-n+2-i)$ for $i=0, \ldots, n-2$, where $c_{i}=(n-1-i)\left(\begin{array}{l}n \\ i\end{array}\right)$.

(b) $\operatorname{Tor}_{n-1}^{S}\left(K, J_{L}: g / J_{L}\right) \cong K(-2 n+2)$.

Proof. It is well known that $S / J_{\tilde{G}}$ is Cohen-Macaulay with the minimal free resolution

$$
0 \rightarrow S^{b_{n-1}}(-n) \rightarrow \cdots \rightarrow S^{b_{n-1-i}}(-n+i) \rightarrow \cdots \rightarrow S^{b_{1}}(-2) \rightarrow S
$$

where $b_{i}=i\left(\begin{array}{c}n \\ i+1\end{array}\right)$. By Lemma 10 (a) and Theorem 7, we have the above statement.

Now we will give the theorem in which we compute all Tor modules of $S / J_{L}: g$.

Theorem 13. With the previous notation we have 
(a) $\operatorname{Tor}_{i}^{S}\left(K, S / J_{L}: g\right) \cong K^{\left(\begin{array}{c}n-1 \\ i\end{array}\right)}(-2 i) \oplus K^{c_{i-1}}(-n+3-i)$ for $i=1, \ldots, n-3$,

(b) $\operatorname{Tor}_{n-2}^{S}\left(K, S / J_{L}: g\right) \cong K^{c_{n-3}}(-2 n+5)$,

(c) $\operatorname{Tor}_{n-1}^{S}\left(K, S / J_{L}: g\right) \cong K^{\left(\begin{array}{c}n-1 \\ 2\end{array}\right)}(-2 n+4)$,

(d) $\operatorname{reg}\left(S / J_{L}: g\right)=n-3$.

Proof. Consider the exact sequence

$$
0 \rightarrow J_{L}: g / J_{L} \rightarrow S / J_{L} \rightarrow S / J_{L}: g \rightarrow 0 .
$$

Let $i<n-2$, then the above exact sequence induces a graded homomorphism of degree zero

$$
\operatorname{Tor}_{i}^{S}\left(K, J_{L}: g / J_{L}\right) \cong K^{c_{i}}(-n+2-i) \rightarrow \operatorname{Tor}_{i}^{S}\left(K, S / J_{L}\right) \cong K^{\left(\begin{array}{c}
n-1 \\
i
\end{array}\right)}(-2 i) .
$$

Therefore it is the zero homomorphism so we have the following isomorphism

$$
\operatorname{Tor}_{i}^{S}\left(K, S / J_{L}: g\right) \cong \operatorname{Tor}_{i}^{S}\left(K, S / J_{L}\right) \oplus \operatorname{Tor}_{i-1}^{S}\left(K, J_{L}: g / J_{L}\right)
$$

Let $i=n-1$, then we get the injection $0 \rightarrow K(-2 n+2) \rightarrow K(-2 n+2)$ which is in fact an isomorphism. So we have the following exact sequence of K-vector spaces.

$$
\begin{gathered}
0 \rightarrow \operatorname{Tor}_{n-1}^{S}\left(K, S / J_{L}: g\right) \rightarrow \operatorname{Tor}_{n-2}^{S}\left(K, J_{L}: g / J_{L}\right) \cong K^{c_{n-2}}(-2 n+4) \rightarrow \operatorname{Tor}_{n-2}^{S}\left(K, S / J_{L}\right) \\
\cong K^{\left(\begin{array}{c}
n-1 \\
1
\end{array}\right)}(-2 n+4) \rightarrow \operatorname{Tor}_{n-2}^{S}\left(K, S / J_{L}: g\right) \rightarrow \operatorname{Tor}_{n-3}^{S}\left(K, J_{L}: g / J_{L}\right) \rightarrow 0 .
\end{gathered}
$$

By Lemma 11 (b) and Theorem 7 we have $\operatorname{Tor}_{n-1}^{S}\left(K, S / J_{L}: g\right) \cong K^{\left(\begin{array}{c}n-1 \\ 2\end{array}\right)}(-2 n+4)$ since $S / J_{L}: g$ is Cohen-Macaulay. By investigating the K-vector space dimension of these modules and $c_{n-2}=\left(\begin{array}{c}n \\ 2\end{array}\right)=\left(\begin{array}{c}n-1 \\ 2\end{array}\right)+\left(\begin{array}{c}n-1 \\ 1\end{array}\right)$, it follows that $\operatorname{Tor}_{n-2}^{S}\left(K, S / J_{L}: g\right) \cong$ $\operatorname{Tor}_{n-3}^{S}\left(K, J_{L}: g / J_{L}\right)$.

Lemma 14. The coefficient of the highest power $t^{n-1}$ of the numerator of the Hilbert series $H\left(S / J_{C}, t\right)$ is $\left(\begin{array}{c}n-1 \\ 2\end{array}\right)-1$.

Proof. If we expand $(1+t)^{n-1}$ in the numerator of $H\left(S / J_{C}, t\right)$ of Theorem 10 (b). The last two terms in the numerator cancels and we get $1+(n-1) t+\cdots+\left(\left(\begin{array}{c}n-1 \\ 2\end{array}\right)-1\right) t^{n-1}$.

Now we are ready to say about all Tor modules of $S / J_{C}$.

Theorem 15. With the previous notation we have

(a) $\operatorname{Tor}_{i}^{S}\left(K, S / J_{C}\right) \cong K^{\left(\begin{array}{l}n \\ i\end{array}\right)}(-2 i) \oplus K^{c_{i-2}}(-n+2-i)$ for $i=1, \ldots, n-2$,

(b) $\operatorname{Tor}_{n-1}^{S}\left(K, S / J_{C}\right) \cong K^{c_{n-3}}(-2 n+3)$,

(c) $\operatorname{Tor}_{n}^{S}\left(K, S / J_{C}\right) \cong K^{\left(\begin{array}{c}n-1 \\ 2\end{array}\right)-1}(-2 n+2)$. 
Proof. Consider the exact sequence

$$
0 \rightarrow\left(S / J_{L}: g\right)(-2) \rightarrow S / J_{L} \rightarrow S / J_{C} \rightarrow 0 .
$$

Let $i<n-1$, then the above exact sequence induces a graded homomorphism of degree zero

$$
\begin{gathered}
\operatorname{Tor}_{i}^{S}\left(K, S / J_{L}: g\right)(-2) \cong K^{\left(\begin{array}{c}
n-1 \\
i
\end{array}\right)}(-2 i-2) \oplus K^{c_{i-1}}(-n+1-i) \\
\rightarrow \operatorname{Tor}_{i}^{S}\left(K, S / J_{L}\right) \cong K^{\left(\begin{array}{c}
n-1 \\
i
\end{array}\right)}(-2 i) .
\end{gathered}
$$

First of all we observe that $\operatorname{reg} S / J_{C}=n-2$ as Therefore it is the zero homomorphism so we have the following isomorphism

$$
\operatorname{Tor}_{i}^{S}\left(K, S / J_{C}\right) \cong \operatorname{Tor}_{i}^{S}\left(K, S / J_{L}\right) \oplus \operatorname{Tor}_{i-1}^{S}\left(K, S / J_{L}: g\right)(-2) .
$$

Let $i=n$, then we have the following exact sequence of K-vector spaces

$$
\begin{aligned}
& 0 \rightarrow \operatorname{Tor}_{n}^{S}\left(K, S / J_{C}\right) \rightarrow \operatorname{Tor}_{n-1}^{S}\left(K, S / J_{L}: g\right)(-2) \cong K^{\left(\begin{array}{c}
n-1 \\
2
\end{array}\right)}(-2 n+2) \rightarrow \operatorname{Tor}_{n-1}^{S}\left(K, S / J_{L}\right) \\
& \cong K(-2 n+2) \rightarrow \operatorname{Tor}_{n-1}^{S}\left(K, S / J_{C}\right) \rightarrow \operatorname{Tor}_{n-2}^{S}\left(K, S / J_{L}: g\right)(-2) \cong K^{c_{n-3}}(-2 n+3) \rightarrow 0 .
\end{aligned}
$$

First of all we observe that $\operatorname{reg} S / J_{C}=n-2$ as follows by the above isomorphisms and the previous exact sequence. Moreover, the exact sequence provides that $\operatorname{Tor}_{n}^{S}\left(K, S / J_{C}\right) \cong$ $K^{x}(-2 n+2)$ for a certain positive integer $x \in \mathbb{N}$. By view of the expression of

$$
n-2=\operatorname{reg} S / J_{C}=\max \left\{j-i \in \mathbb{Z} \mid \beta_{i, j}\left(S / J_{C}\right) \neq 0\right\}
$$

it follows that $\beta_{i, j}\left(S / J_{C}\right)=0$ for all $j-i>n-2$ and all $i>n$. Therefore, computing the Hilbert series $H\left(S / J_{C}, t\right)$ by the minimal free resolution (see Lemma 5 ) has the form

$$
H\left(S / J_{C}, t\right)=p(t) /(1-t)^{2 n},
$$

where $p(t)$ is a polynomial of degree $2 n-2$ with leading term $(-1)^{n-1} \beta_{n, 2 n-2}\left(S / J_{C}\right) t^{2 n-2}$. Comparing it with the expansion of the Hilbert series as given in Lemma 14 it follows that $\operatorname{Tor}_{n}^{S}\left(K, S / J_{C}\right) \cong K^{\left(\begin{array}{c}n-1 \\ 2\end{array}\right)-1}(-2 n+2)$ which further implies that $\operatorname{Tor}_{n-1}^{S}\left(K, S / J_{C}\right) \cong$ $K^{c_{n-3}}(-2 n+3)$.

As a consequence of Theorem 15 we are now able to describe the explicit Betti numbers of the binomial edge ideal of a cycle.

Corollary 16. Let $S / J_{C}$ be binomial edge ideal of cycle on vertex set $[n]$. Then the $\operatorname{reg}\left(S / J_{C}\right)=n-2$ and the Betti diagram of the $S / J_{C}$ looks like the following:

\begin{tabular}{c|ccccccc} 
& 0 & 1 & 2 & $\cdots$ & $n-2$ & $n-1$ & $n$ \\
\hline 0 & 1 & 0 & 0 & $\cdots$ & 0 & 0 & 0 \\
1 & 0 & $\beta_{1,2}$ & 0 & $\cdots$ & 0 & 0 & 0 \\
2 & 0 & 0 & $\beta_{2,4}$ & $\cdots$ & 0 & 0 & 0 \\
$\vdots$ & $\vdots$ & $\vdots$ & $\vdots$ & $\ddots$ & $\vdots$ & $\vdots$ & $\vdots$ \\
$n-2$ & 0 & 0 & $\beta_{2, n}$ & $\cdots$ & $\beta_{n-2,2 n-4}$ & $\beta_{n-1,2 n-3}$ & $\beta_{n, 2 n-2}$
\end{tabular}


where the Betti numbers in the diagonal are

$$
\beta_{i, 2 i}=\left(\begin{array}{c}
n \\
i
\end{array}\right), \text { if } i=0, \ldots, n-3
$$

and the last row of Betti diagram is

$$
\beta_{i, n-2+i}= \begin{cases}c_{i-2}, & \text { if } i=2, \ldots, n-3 \\
\left(\begin{array}{c}
n \\
2
\end{array}\right)+c_{n-4}, & \text { if } i=n-2 \\
c_{n-3}, & \text { if } i=n-1 \\
\left(\begin{array}{c}
n-1 \\
2
\end{array}\right)-1, & \text { if } i=n .\end{cases}
$$

Proof. It follows from Theorem 15.

Corollary 17. Let $G$ be any arbitrary graph on vertex set $[n]$. Let $C$ denote a cycle on maximal $k$ vertices as an induced subgraph. Then $\operatorname{reg}\left(S / J_{G}\right) \geqslant k-2$ and $\beta_{i, j}\left(S / J_{G}\right) \geqslant$ $\beta_{i, j}\left(S / J_{C}\right)$, where the values of $\beta_{i, j}\left(S / J_{C}\right)$ are those of Corollary 16 for $n=k$.

Proof. It follows from Theorem 8 and Corollary 16.

Remark 18. In case $G$ has a cycle $C$ on maximal $k$ vertices as an induced subgraph it has also a line $L$ on $k-1$ vertices. That is, the lower bound $k-2 \leqslant \operatorname{reg}\left(S / J_{G}\right)$ is not better than that of [5]. The advantage of Corollary 17 is that it provides the non-vanishing of certain Betti numbers different from those of $\beta_{i, j}\left(S / J_{L}\right)$.

\section{Betti Numbers of the binomial edge ideal of $\mathcal{T}_{3}$ and $\mathcal{G}_{3}$}

The clique complex of a graph was used by many authors (see e.g. [3],[7] and [8]) to study binomial edge ideals. Here in the following we introduce this nice concept.

Definitions 19. Let $G$ be a simple graph on vertex set $[n]$.

1. A clique of $G$ is a subset $W$ of $[n]$ such that each vertex in $W$ is connected to any other vertex in $W$ by an edge of $G$. In other words it is a complete subgraph of $G$.

2. A maximal clique is a clique that is not a subset of a larger clique.

3. The clique complex $\Delta(G)$ of $G$ is a simplicial complex whose facets are the maximal cliques of $G$.

4. A vertex $j \in[n]$ is called free vertex if it belongs to only one facet of $\Delta(G)$.

For example, in a complete graph all vertices are free vertices, and in any graph the vertices of degree 1 are free vertices, while cycle of length $>3$ has no free vertices.

The following proposition whose proof can be found in [7, Proposition 2.1] is important for us. 
Proposition 20. Let $G$ be a simple graph on vertex set $[n]$. Let $\Delta(G)$ is clique complex of $G$ and $j \in[n]$ be a vertex of $G$. Then the following conditions are equivalent:

1. $j$ is a free vertex of $\Delta(G)$.

2. $j \notin T$ for all $T \subseteq[n]$ such that $c(T \backslash\{i\})<c(T)$ for each $i \in T$.

The following lemma tells us the importance of the free vertex.

Lemma 21. Let $G$ be the graph on vertex set $[n]$ with at least one free vertex and $J_{G}$ be its binomial edge ideal. Chose one of its free vertex and label it by $n$. Let $J_{G^{\prime}}$ denotes the binomial edge ideal of the graph $G^{\prime}$ by attaching the edge $\{n, n+1\}$ to the graph $G$. Then $f=x_{n} y_{n+1}-x_{n+1} y_{n}$ is regular on $S^{\prime} / J_{G}$ where $S^{\prime}=S\left[x_{n+1}, y_{n+1}\right]$, and we have the following exact sequence of $S^{\prime}$-modules

$$
0 \rightarrow S^{\prime} / J_{G}(-2) \stackrel{f}{\rightarrow} S^{\prime} / J_{G} \rightarrow S^{\prime} / J_{G^{\prime}} \rightarrow 0 .
$$

where $J_{G^{\prime}}=\left(J_{G}, f\right)$

Proof. Since $n$ is free vertex therefore by Proposition 20 and Lemma 3 (c) $x_{n}, y_{n} \notin P_{T}(G)$ for all $P_{T}(G) \in \operatorname{Ass}\left(S / J_{G}\right)$, and hence $f \notin P_{T}(G)$ for all $P_{T}(G) \in \operatorname{Ass}\left(S^{\prime} / J_{G}\right)$. Therefore $f$ is not a zero divisor in $S^{\prime} / J_{G}$ and is regular, and one obtains the above exact sequence.

Definition 22. Let $\mathcal{T}_{3}$ be the collection of graphs such that for all $G \in \mathcal{T}_{3}$ we have

$$
V(G)=\left\{u_{1}, \ldots, u_{r}, v_{1}, \ldots, v_{s}, w_{1}, \ldots, w_{t}\right\}
$$

with $r \geqslant 2, s \geqslant 1, t \geqslant 1$ and edge set

$$
\begin{aligned}
E(G)= & \left\{\left\{u_{i}, u_{i+1}\right\}: i=1, \ldots, r-1\right\} \cup\left\{\left\{v_{i}, v_{i+1}\right\}: i=1, \ldots, s-1\right\} \\
& \cup\left\{\left\{w_{i}, w_{i+1}\right\}: i=1, \ldots, t-1\right\} \cup\left\{\left\{u_{1}, v_{1}\right\},\left\{u_{1}, w_{1}\right\}\right\} .
\end{aligned}
$$

Note that any $G \in \mathcal{T}_{3}$ is a tree which have at most one vertex of degree 3 .

Example 23. Consider the simplest example of the graph in $\mathcal{T}_{3}$ as shown in Figure 1.

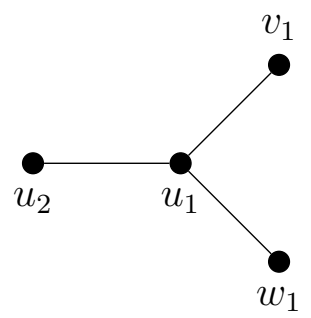

Figure 1 
It is easy to see that $u_{2}, v_{1}$ and $w_{1}$ are free vertices of $G$. Its binomial edge ideal has the following Betti diagram

\begin{tabular}{l|llll} 
& 0 & 1 & 2 & 3 \\
\hline 0 & 1 & 0 & 0 & 0 \\
1 & 0 & 3 & 0 & 0 \\
2 & 0 & 0 & 4 & 2
\end{tabular}

and it has the following Hilbert series

$$
H\left(S / J_{G}, t\right)=\frac{1}{(1-t)^{6}}\left(1+2 t-2 t^{3}\right) .
$$

It is known from [13, Corollary 3.5] that any $G \in \mathcal{T}_{3}$ on the vertex set $[n]$, the ring $S / J_{G}$ is approximately Cohen-Macaulay $\operatorname{ring}$ of $\operatorname{dim}\left(S / J_{G}\right)=n+2$.

Remark 24. We use computer algebra system CoCoA [2] for the computations of some arithmetic invariants of $S / J_{G}$ in Example 23 and 26.

Definition 25. (see [8]) $\mathcal{G}_{3}$ be the collection of graphs such that for all $G \in \mathcal{G}_{3}$ we have

$$
V(G)=\left\{u_{1}, \ldots, u_{r}, v_{1}, \ldots, v_{s}, w_{1}, \ldots, w_{t}\right\}
$$

with $r \geqslant 1, s \geqslant 1, t \geqslant 1$ and edge set

$$
\begin{gathered}
E(G)=\left\{\left\{u_{i}, u_{i+1}\right\}: i=1, \ldots, r-1\right\} \cup\left\{\left\{v_{i}, v_{i+1}\right\}: i=1, \ldots, s-1\right\} \\
\cup\left\{\left\{w_{i}, w_{i+1}\right\}: i=1, \ldots, t-1\right\} \cup\left\{\left\{u_{1}, v_{1}\right\},\left\{u_{1}, w_{1}\right\},\left\{v_{1}, w_{1}\right\}\right\} .
\end{gathered}
$$

Example 26. The simplest example of the graph in $\mathcal{G}_{3}$ is the complete graph on the vertex set $u_{1}, v_{1}$ and $w_{1}$ and all of them are free vertices of $G$. Its binomial edge ideal has the following Betti diagram

\begin{tabular}{l|lll} 
& 0 & 1 & 2 \\
\hline 0 & 1 & 0 & 0 \\
1 & 0 & 3 & 2
\end{tabular}

and it has the following Hilbert series

$$
H\left(S / J_{G}, t\right)=\frac{1}{(1-t)^{4}}(1+2 t) .
$$

It is known from [8, Proposition 2.5] that any $G \in \mathcal{G}_{3}$ on vertex set $[n]$ is CohenMacaulay ring of $\operatorname{dim}\left(S / J_{G}\right)=n+1$. Note that the $\operatorname{proj} \operatorname{dim}\left(S / J_{G}\right)=n-1$ for any $G \in \mathcal{T}_{3} \cup \mathcal{G}_{3}$ on vertex set $[n]$ as it will be shown in the following result.

Theorem 27. Let $G$ be the graph on vertex set $[n]$ and $G \in \mathcal{T}_{3} \cup \mathcal{G}_{3}$. Let $J_{G}$ denotes its binomial edge ideal then the $\operatorname{reg}\left(S / J_{G}\right)=n-2$ and the Betti diagram of the $S / J_{G}$ looks like the following: 


\begin{tabular}{c|cccccccc} 
& 0 & 1 & 2 & 3 & 4 & $\cdots$ & $n-2$ & $n-1$ \\
\hline 0 & 1 & 0 & 0 & 0 & 0 & $\cdots$ & 0 & 0 \\
1 & 0 & $\beta_{1,2}$ & $\beta_{2,3}$ & 0 & 0 & $\cdots$ & 0 & 0 \\
2 & 0 & 0 & $\beta_{2,4}$ & $\beta_{3,5}$ & 0 & $\cdots$ & 0 & 0 \\
3 & 0 & 0 & 0 & $\beta_{3,6}$ & $\beta_{4,7}$ & $\cdots$ & 0 & 0 \\
$\vdots$ & $\vdots$ & $\vdots$ & $\vdots$ & $\vdots$ & $\ddots$ & $\ddots$ & $\vdots$ & $\vdots$ \\
$n-3$ & 0 & 0 & 0 & 0 & 0 & $\ddots$ & $\ddots$ & 0 \\
$n-2$ & 0 & 0 & 0 & 0 & 0 & $\cdots$ & $\beta_{n-2,2 n-4}$ & $\beta_{n-1,2 n-3}$
\end{tabular}

Proof. We want to prove the claim on the Betti table by induction on $n$. For $n=3$ or $n=4$ it is true (see Example 23 and 26). Now let us assume that the statement is true for $n$. We use the notations of Lemma 21 because in these classes we inductively go from graph $G$ on $n$ vertices to graph $G^{\prime}$ on $n+1$ vertices by adding an edge $\{n, n+1\}$ with the assumption that $n$ is free vertex. Let $F_{\bullet}$ be the minimal free resolution for $S / J_{G}$ then $F_{\bullet} \otimes_{S} S^{\prime}$ is minimal free resolution for $S^{\prime} / J_{G}$ and hence they have the same Betti numbers. To this end we note that

$$
\operatorname{Tor}_{i}^{S}\left(K, S / J_{G}\right) \cong \operatorname{Tor}_{i}^{S^{\prime}}\left(K, S^{\prime} / J_{G}\right)
$$

Hence we have the following isomorphism restricted to degree $i+j$.

$$
\operatorname{Tor}_{i}^{S^{\prime}}\left(K, S^{\prime} / J_{G}\right)_{i+j} \cong \operatorname{Tor}_{i}^{S}\left(K, S / J_{G}\right)_{i+j} \cong K^{\beta_{i, i+j}}(-(i+j))
$$

Now consider the exact sequence of Lemma 21

$$
0 \rightarrow S^{\prime} / J_{G}(-2) \stackrel{f}{\rightarrow} S^{\prime} / J_{G} \rightarrow S^{\prime} / J_{G^{\prime}} \rightarrow 0 .
$$

The first two modules of the above exact sequence are the same modules with the shift of degree 2. Next we want to show that the map

$$
\phi_{i}: \operatorname{Tor}_{i}^{S^{\prime}}\left(K, S^{\prime} / J_{G}(-2)\right) \rightarrow \operatorname{Tor}_{i}^{S^{\prime}}\left(K, S^{\prime} / J_{G}\right) .
$$

is the zero map. To this end it will be enough to show that

$$
\left[\phi_{i}\right]_{j}: \operatorname{Tor}_{i}^{S^{\prime}}\left(K, S^{\prime} / J_{G}\right)_{i+j-2} \rightarrow \operatorname{Tor}_{i}^{S^{\prime}}\left(K, S^{\prime} / J_{G}\right)_{i+j} .
$$

is zero for all $j$. Now suppose that

$$
0 \neq \operatorname{Tor}_{i}^{S^{\prime}}\left(K, S^{\prime} / J_{G}\right)_{i+j-2} \cong \operatorname{Tor}_{i}^{S}\left(K, S / J_{G}\right)_{i+j-2}
$$

By induction hypothesis for $n$ it turns out that $(i, j-2)$ is either $(i, i)$ or $(i, i-1)$. In the first case, that is $j-2=i$, the target of $\left[\phi_{i}\right]_{j}$ is

$$
\operatorname{Tor}_{i}^{S^{\prime}}\left(K, S^{\prime} / J_{G}\right)_{2 i+2} \cong \operatorname{Tor}_{i}^{S}\left(K, S / J_{G}\right)_{2 i+2}=0
$$


In the second case, that is $j-2=i-1$, the target of $\left[\phi_{i}\right]_{j}$ is

$$
\operatorname{Tor}_{i}^{S^{\prime}}\left(K, S^{\prime} / J_{G}\right)_{2 i+1} \cong \operatorname{Tor}_{i}^{S}\left(K, S / J_{G}\right)_{2 i+1}=0
$$

Now suppose that the target of $\left[\phi_{i}\right]_{j}$ namely $\operatorname{Tor}_{i}^{S^{\prime}}\left(K, S^{\prime} / J_{G}\right)_{i+j}$ is non-zero. Again by induction hypothesis for $n$ it follows that $(i, j)$ is either $(i, i)$ or $(i, i-1)$. In the first case, that is $j=i$, the source of $\left[\phi_{i}\right]_{j}$ is

$$
\operatorname{Tor}_{i}^{S^{\prime}}\left(K, S^{\prime} / J_{G}\right)_{2 i-2} \cong \operatorname{Tor}_{i}^{S}\left(K, S / J_{G}\right)_{2 i-2}=0
$$

In the second case, that is $j=i-1$, the source of $\left[\phi_{i}\right]_{j}$ is

$$
\operatorname{Tor}_{i}^{S^{\prime}}\left(K, S^{\prime} / J_{G}\right)_{2 i-3} \cong \operatorname{Tor}_{i}^{S}\left(K, S / J_{G}\right)_{2 i-3}=0
$$

This completes the proof for $\phi_{i}$ is the zero map. Therefore the short exact sequence induces an isomorphism

$$
\operatorname{Tor}_{i}^{S^{\prime}}\left(K, S^{\prime} / J_{G^{\prime}}\right) \cong \operatorname{Tor}_{i}^{S^{\prime}}\left(K, S^{\prime} / J_{G}\right) \oplus \operatorname{Tor}_{i-1}^{S^{\prime}}\left(K, S^{\prime} / J_{G}(-2)\right)
$$

In order to complete the inductive step we have to show that $\operatorname{Tor}_{i}^{S^{\prime}}\left(K, S^{\prime} / J_{G^{\prime}}\right)_{i+j}$ is zero for all $(i, j)$ different of $(i, i)$ and $(i, i-1)$. This follows because of

$$
\operatorname{Tor}_{i}^{S^{\prime}}\left(K, S^{\prime} / J_{G^{\prime}}\right)_{i+j} \cong \operatorname{Tor}_{i}^{S^{\prime}}\left(K, S^{\prime} / J_{G}\right)_{i+j} \oplus \operatorname{Tor}_{i-1}^{S^{\prime}}\left(K, S^{\prime} / J_{G}\right)_{i+j-2} .
$$

Note that if $(i, j) \neq(i, i-1)$ and $(i, j) \neq(i, i)$, then $(i-1, j-1) \neq(i-1, i-2)$ and $(i-1, j-1) \neq(i-1, i-1)$. Moreover we get

$$
\beta_{i, 2 i}\left(S^{\prime} / J_{G^{\prime}}\right)=\beta_{i, 2 i}\left(S / J_{G}\right)+\beta_{i-1,2 i-2}\left(S / J_{G}\right) .
$$

and

$$
\beta_{i, 2 i-1}\left(S^{\prime} / J_{G^{\prime}}\right)=\beta_{i, 2 i-1}\left(S / J_{G}\right)+\beta_{i-1,2 i-3}\left(S / J_{G}\right) .
$$

Hence any Betti number of $S^{\prime} / J_{G^{\prime}}$ is the sum of consecutive Betti numbers of $S / J_{G}$ of its same diagonal. This completes the inductive step.

The recursion formulas for the Betti numbers at the end of the proof might be used for an explicit computation of them. We will follow here a different approach using Hilbert series.

Lemma 28. With the notations of Lemma 21, we have

$$
H\left(S^{\prime} / J_{G^{\prime}}, t\right)=\left(1-t^{2}\right) H\left(S^{\prime} / J_{G}, t\right) .
$$

Proof. The desired identity of Hilbert series follows from the exact sequence

$$
0 \rightarrow S^{\prime} / J_{G}(-2) \stackrel{f}{\rightarrow} S^{\prime} / J_{G} \rightarrow S^{\prime} / J_{G^{\prime}} \rightarrow 0
$$

by using the additivity of Hilbert series on short exact sequences. 
Lemma 29. Let $G$ be a graph on vertex set $[n]$ and $J_{G}$ be its binomial edge ideal.

(a) Let $G \in \mathcal{T}_{3}$ then the Hilbert series is

$$
H\left(S / J_{G}, t\right)=\frac{1}{(1-t)^{n+2}}\left(1+2 t-2 t^{3}\right)(1+t)^{n-4} \text { for } n>3 .
$$

(b) Let $G \in \mathcal{G}_{3}$ then the Hilbert series is

$$
H\left(S / J_{G}, t\right)=\frac{1}{(1-t)^{n+1}}(1+2 t)(1+t)^{n-3} \text { for } n>2 .
$$

Proof. We will prove (a) by induction on $n$. For $n=4$ it is true, see Example 23. Suppose the claim is true for $n$. That is,

$$
H\left(S^{\prime} / J_{G}, t\right)=\frac{1}{(1-t)^{n+4}}\left(1+2 t-2 t^{3}\right)(1+t)^{n-4} .
$$

Now by previous lemma we have

$$
H\left(S^{\prime} / J_{G^{\prime}}, t\right)=\frac{1}{(1-t)^{n+3}}\left(1+2 t-2 t^{3}\right)(1+t)^{n-3}
$$

as required.

Similar arguments might be used in order to calculate the Hilbert series in (b).

Theorem 30. Let $G$ be a graph on vertex set $[n]$ and $J_{G}$ be its binomial edge ideal.

(a) Let $G \in \mathcal{T}_{3}$ then the Betti numbers for $S / J_{G}$ are:

$$
\beta_{i, j}= \begin{cases}\left(\begin{array}{c}
n-4 \\
i
\end{array}\right)+3\left(\begin{array}{c}
n-4 \\
i-1
\end{array}\right)+4\left(\begin{array}{c}
n-4 \\
i-2
\end{array}\right), & \text { if } j=2 i \text { and } i=0, \ldots, n-2 ; \\
2\left(\begin{array}{c}
n-4 \\
i-3
\end{array}\right), & \text { if } j=2 i-1 \text { and } i=3, \ldots, n-1 ; \\
0, & \text { otherwise }\end{cases}
$$

(b) Let $G \in \mathcal{G}_{3}$ then the Betti numbers for $S / J_{G}$ are:

$$
\beta_{i, j}= \begin{cases}3\left(\begin{array}{c}
n-3 \\
i-1
\end{array}\right)+\left(\begin{array}{c}
n-3 \\
i
\end{array}\right), & \text { if } j=2 i \text { and } i=0, \ldots, n-2 ; \\
2\left(\begin{array}{c}
n-3 \\
i-2
\end{array}\right), & \text { if } j=2 i-1 \text { and } i=2, \ldots, n-1 ; \\
0, & \text { otherwise. }\end{cases}
$$

Proof. By Lemma 5 and the structure of the Betti table (see in the above Theorem 27) provides the following formula

$$
H\left(S / J_{G}, t\right)=\frac{1}{(1-t)^{2 n}}\left(\sum_{i=0}^{n-2}(-1)^{i} \beta_{i, 2 i} t^{2 i}+\sum_{i=2}^{n-1}(-1)^{i} \beta_{i, 2 i-1} t^{2 i-1}\right) .
$$

and after comparing the Hilbert series of Lemma 29 (a) and by making a few simple computations we have the formulas for the Betti numbers in (a). Similar computation using the Hilbert series of Lemma 29 (b) gives the Betti numbers in (b). 
Corollary 31. Let $G$ be any arbitrary graph on vertex set $[n]$. Suppose that $G$ has an induced subgraph $H \in \mathcal{T}_{3} \cup \mathcal{G}_{3}$ on $k$ vertices. Then $\operatorname{reg}\left(S / J_{G}\right) \geqslant k-2$.

Proof. It is an easy consequence of Theorem 8 and 27.

Remark 32. Let $G$ denote a graph with the largest $k$ such that $G$ has an induced subgraph $H \in \mathcal{T}_{3} \cup \mathcal{G}_{3}$. Then it has also a line $L$ as an induced subgraph with $\ell=\max \{s+t+1, r+$ $t, r+s\}$ resp. $\ell=\max \{r+s, r+t, s+t\}$ vertices. In general $k>\ell$, so that the lower bound for the Castelnuovo-Mumford regularity in Corollary 31 improves those of [5].

Acknowledgements: The authors are grateful to the reviewer for suggestions to improve the presentation of the manuscript.

\section{References}

[1] W. Bruns, J. Herzog. Cohen-Macaulay Rings, Cambridge University Press, 1993.

[2] The CoCoA Team, CoCoA. A system for doing Computations in Commutative Algebra, available at http://cocoa.dima.unige.it.

[3] V. Ene, J. Herzog and T. Hibi. Cohen-Macaulay Binomial edge ideals. Nagoya Math. J. 204 (2011) 57-68.

[4] J. Herzog, T. Hibi, F. Hreinsdotir, T. Kahle, J, Rauh. Binomial edge ideals and conditional independence statements. Adv. Appl. Math. 45 (2010) 317-333.

[5] K. Matsuda and S. Murai. Regularity bounds for binomial edge ideals, J. Comm. Algebra, 5 (2013), 141-149.

[6] M. Ohtani. Graphs and ideals generated by some 2-minors, J. Comm. Algebra., 39 (2011), 905-917.

[7] A. Rauf, G. Rinaldo. Construction of Cohen-Macaulay binomial edge ideals, Accepted in J. Comm. Algebra.

[8] G. Rinaldo. Cohen-Macaulay binomial edge ideals of small deviation, Bull. Math. Soc. Sci. Math. Roumanie Tome 56(104) No. 4, 2013, 497-503.

[9] S. Saeedi, D. Kiani. Binomial edge ideals of graphs, Electronic journal of combinatorics, 19 (2) (2012).

[10] P. Schenzel. On The Use of Local Cohomology in Algebra and Geometry. In: Six Lectures in Commutative Algebra, Proceed. Summer School on Commutative Algebra at Centre de Recerca Matemàtica, (Ed.: J. Elias, J. M. Giral, R. M. Miró-Roig, S. Zarzuela), Progr. Math. 166, pp. 241-292, Birkhäuser, 1998.

[11] P. Schenzel, S. Zafar. Algebraic properties of the binomial edge ideal of complete bipartite graph. To appear in An. St. Univ. Ovidius Constanta, Ser. Mat.

[12] R. H. Villarreal. Monomial Algebras, New York: Marcel Dekker Inc. (2001).

[13] S. Zafar. On approximately Cohen-Macaulay binomial edge ideal, Bull. Math. Soc. Sci. Math. Roumanie, 55(103) (2012), 429-442. 